\section{A Preliminary Study on Radiation Shielding Using Martian Magnetic Anomalies}

\author{
Kazuma Emoto ${ }^{1 *}$, Yoshinori Takao ${ }^{2}$, and \\ Hitoshi Kuninaka $^{3}$ \\ ${ }^{1}$ Department of Systems Integration, Yokohama \\ National University, 79-5, Tokiwadai, Hodogaya-ku, \\ Yokohama 240-8501, Japan \\ ${ }^{2}$ Division of Systems Research, Yokohama National \\ University, 79-5, Tokiwadai, Hodogaya-ku, \\ Yokohama 240-8501, Japan \\ ${ }^{3}$ Institute of Space and Astronautical Science, Japan \\ Aerospace Exploration Agency, 3-1-1, Yoshinodai, \\ Chuo-ku, Sagamihara 252-5210, Japan
}

\begin{abstract}
We propose radiation shielding using Martian magnetic anomalies to protect human crews on the Martian surface. We have simulated the trajectories of energetic protons using the Buneman-Boris method to measure how magnetic anomalies affect the impact rate on the Martian surface. Protons from the west can be completely eliminated, while those from the east are concentrated on the area between the magnetic poles. This would mean crews would need to concern themselves about radiation from the vertex and east only. A Martian magnetic anomaly can therefore be used to realize continuous and efficient radiation shielding.
\end{abstract}

\section{Introducion}

Manned Mars exploration and migration missions are currently being planned by many governments and private organizations (Smith et al., 2017; Musk, 2017). However, long-term human activity on Mars will entail many problems. One particular-problem is human exposure to harsh radiation in the form of solar energetic particles (SEPs) and galactic cosmic rays (GCRs) caused by the lack of intrinsic magnetic field on Mars (Acuña et al., 1998).

As SEPs and GCRs penetrate to the Martian surface, crews would not be able to live or explore continuously outside of an artificial habitat. They would have to stay in such a habitat or under the ground to protect their health from radiation exposure. Continuous radiation shielding will be necessary for human activities on the Martian surface to occur safely.

Received: December 5, 2017; Accepted: March 5, 2018

*To whom correspondence should be addressed:

Tel/Fax.: +81-(0)45-339-3889;

E-mail: kazuma-emoto-vh@ynu.jp
To protect crews from SEPs and GCRs, we focus here on the possibility of using the Martian magnetic anomalies studied in detail by the Mars Global Surveyor (Acuña et al., 1998, 1999, 2001; Langlais et al., 2004; Langlais and Quesnel, 2008; Mitchell et al., 2007; Purucker et al., 2000). In the southern hemisphere and at a longitude of approximately $180^{\circ}$, strong and linear magnetic poles are spread alternately. As the trajectories of energetic particles are altered by the Lorentz force, these particles are not expected to hit the ground between the magnetic poles, leaving regions where crews could be naturally protected from radiation exposure. Although a magnetic anomaly would not completely block energetic particles, even partial radiation shielding can be effective, because it removes the need to shield all directions around the habitat. Thus, such anomalies could serve as useful aids in shielding crews from dangerous energetic particles.

Energetic particles actually collide with the atmosphere and produce secondary particles by nuclear reactions. However, the effect of the atmosphere is smaller than that of magnetic anomalies because of the high altitude and the low density in the southern hemisphere. The density of the atmosphere in the southern hemisphere is less than half that in the northern hemisphere since the difference of the altitude between the southern and northern hemisphere is several kilometers and the scale height of the Martian atmosphere is about $11 \mathrm{~km}$. Hence, focusing the effect of magnetic anomalies only is more appropriate than that of the atmosphere for a preliminary study on radiation shielding.

We investigate how well a Martian magnetic anomaly can provide shielding against energetic particles coming from different directions using a numerical simulation. The main objective in this study is the quick estimation of the radiation shielding using Martian magnetic anomalies. We conduct simulations with a simple magnetic field neglecting collisions with the atmosphere on the Cartesian coordinates. In addition, we focus on the number of particles reached on the Martian surface to understand clearly the effect of the magnetic anomaly and do not calculate their radiation doses.

\section{Materials and Methods}

We simulate the trajectories of energetic particles entering a Martian magnetic anomaly to determine the impact of the anomaly on the amount of particles reaching the surface. We focus on longitudes of $160-200^{\circ}$ and latitudes of $40-60^{\circ}$, where the uniform linear shape of the radial magnetic field is observed (Acuña et al., 1998, 1999, 2001; Langlais et al., 2004; Langlais and Quesnel, 2008; Mitchell et al., 2007; Purucker et al., 2000).

Figure 1 shows the three-dimensional Cartesian model used. We use simple Cartesian coordinates with a linear banded magnetic field instead of actual spherical coordinates with complex magnetic anomalies to estimate quickly. We consider only motions of particles and the magnetic field in the calculation area. The size of the calculation area is $5300 \times 2000 \times 7000 \mathrm{~km}^{3}$. The area 


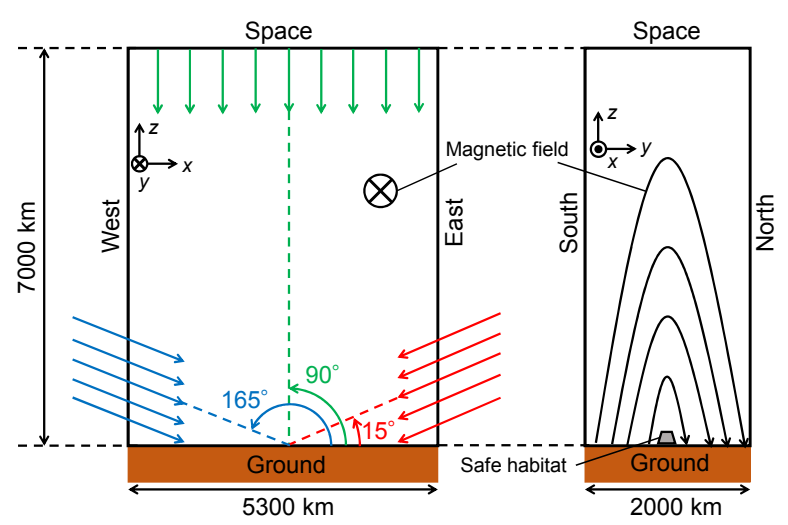

Fig. 1. The three-dimensional Cartesian model used for our numerical simulations. We simulate the motions of SEPs with three incident angles of $15^{\circ}, 90^{\circ}$, and $165^{\circ}$.

around a latitude of $1000 \mathrm{~km}$ is expected to be a safe habitat, where crews would be surrounded by magnetic field lines. In this paper, we simulate the motion of SEPs with three incident angles: $15^{\circ}, 90^{\circ}$, and $165^{\circ}$.

As we wish to focus on the effects of magnetic anomalies, it is assumed that only the Lorentz force is exerted on particles and collisions within the atmosphere are not taken into account. The relativistic equation of motion for such a particle is described as

$$
\frac{d(\gamma m v)}{d t}=q v \times \boldsymbol{B},
$$

where $y$ is the Lorentz factor, $m$ is the mass of the particle, $t$ is the time, $v$ is the velocity of the particle, $q$ is the charge of the particle, and $\boldsymbol{B}$ is the magnetic field applied to the particle. The Lorentz factor $y$ is written as

$$
y=\frac{1}{\sqrt{1-v^{2} / c^{2}}},
$$

where $c$ is the speed of light. To solve Eq. (1), we used the relativistic Buneman-Boris method (Birdsall and Langdon, 1985).

Using uniform random numbers, initial particle positions are assigned on the east, space, and west faces of the calculation area with initial incident angles of $15^{\circ}, 90^{\circ}$, and $165^{\circ}$, respectively. From relativistic theory, a relation between the energy $E$ and the velocity $v$ can be given as

$$
E=\frac{m c^{2}}{\sqrt{1-v^{2} / c^{2}}}-m c^{2}
$$

Solving for the velocity $v$, Eq. (3) is rewritten as

$$
v=\sqrt{c^{2}\left(1-\left(\frac{m c^{2}}{E+m c^{2}}\right)^{2}\right)}
$$

which gives the initial particle velocities for each initial energy. For simplicity, the initial north-south velocities of the particles are set to zero.

We calculated a two-dimensional magnetic field on the altitude-latitude cross-section, which is equivalent to the Martian magnetic anomaly based on observations by the Mars Global Surveyor (Acuña et al., 1998, 1999,
Table 1. Calculation conditions.

\begin{tabular}{ll}
\hline Parameters & Values \\
\hline Calcuation area & $5300 \times 2000 \times 7000 \mathrm{~km}^{3}$ \\
Cell length & $1 \mathrm{~km}$ \\
Particles & Protons \\
Initial energy & $1,10,100 \mathrm{MeV}$, and $1 \mathrm{GeV}$ \\
Initial incident angle & $15^{\circ}, 90^{\circ}$, and $165^{\circ}$ \\
Time step & $1.0 \times 10^{-5} \mathrm{~s}(1,10 \mathrm{MeV})$ or $1.0 \times 10^{-6} \mathrm{~s}$ \\
& $(100 \mathrm{MeV}, 1 \mathrm{GeV})$ \\
\hline
\end{tabular}

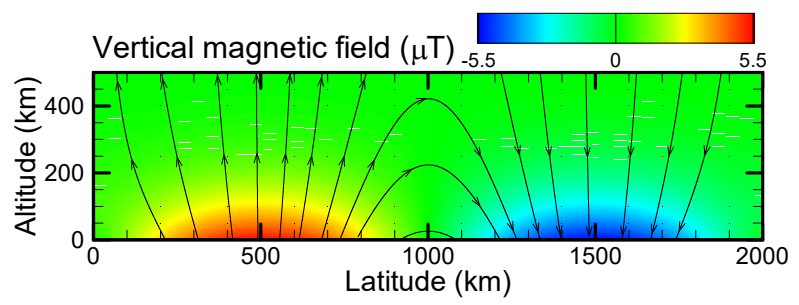

Fig. 2. The magnetic field used for the particle simulation. The altitude is limited to $\mathbf{0}-500 \mathrm{~km}$ to focus on the magnetic field near the ground. For simplicity, we assume a two-dimensional magnetic field on the altitude-latitude cross-section, where the longitudinal magnetic field is zero. The color map shows the intensity of the vertical magnetic field in Tesla, and the arrows represent the magnetic field lines.

2001; Langlais et al., 2004; Langlais and Quesnel, 2008; Mitchell et al., 2007; Purucker et al., 2000). Assuming the magnetic anomaly obtained with the calculation, we simulated particle trajectories and counted the number of particles hitting the surface. Table 1 shows a summary of the calculation conditions. Protons are the only particles considered, as they account for approximately $95 \%$ of SEPs (Sakurai, 1974). It is assumed that the particles have an initial energy of $1 \mathrm{MeV}-1 \mathrm{GeV}$, which is the dominant range of SEP energies and significantly detrimental to human health. Time steps are optimized according to the initial velocities of the protons for each energy.

We use the results of our calculation to plot the number of particles reaching the Martian surface. The number of particle hits is normalized against the value obtained without magnetic field to clarify the effect of the radiation shielding. The radiation exposure is decreased by the effects of the shielding when the normalized particle hits are less than one, and increased when it is greater than one. Normalized particle hits of zero implies the complete shielding of energetic particles.

\section{Results}

Figure 2 shows the altitude-latitude distribution of the magnetic field. The color map shows the intensity of the vertical magnetic field in Tesla, with arrows representing the magnetic field lines. The vertical magnetic field is equal to the observations by the Mars Global Surveyor. Using the magnetic field shown in Fig. 2, we simulated particle trajectories and counted the number of particles 
hitting the surface.

Figure 3 shows the distributions of the normalized particle hits under each condition. The right and left ends of the distributions should be neglected, owing to peculiar results caused by protons injected at low altitudes. When the incident angle is $15^{\circ}$, protons concentrate around a latitude of $1000 \mathrm{~km}$ with an energy range of $1 \mathrm{MeV}-1 \mathrm{GeV}$. At a latitude of $1000 \mathrm{~km}$, protons from the east hit tens of times as often as they would without the Martian magnetic anomaly, and the radiation exposure from SEPS

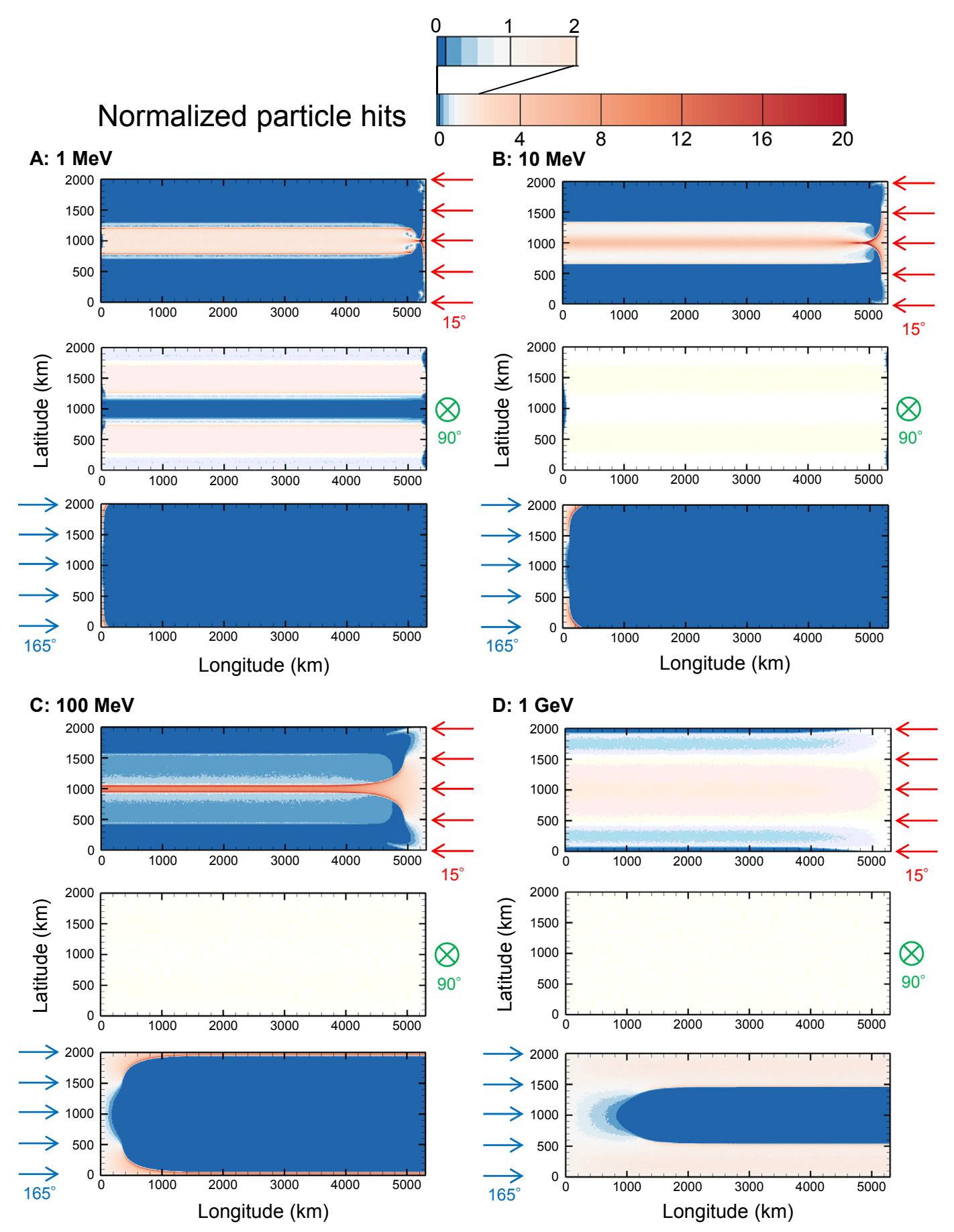

Fig. 3. Distributions of normalized particle hits with initial energies of (A) $1 \mathrm{MeV}$, (B) $10 \mathrm{MeV}$, (C) $100 \mathrm{MeV}$, and (D) $1 \mathrm{GeV}$. Three incident angles for the particles are used: $15^{\circ}, 90^{\circ}$, and $165^{\circ}$. The number of protons is normalized relative to that in the absense of a magnetic field. The radiation exposure is decreased when the normalized particle hits are less than one, and increased when they are greater than one. Normalized particle hits of zero indicate complete shielding from energetic particles. The right and left ends of the distributions should be neglected owing to peculiar results caused by protons injected at low altitudes. 
increases significantly. When the incident angle is $90^{\circ}$, the protons are somewhat shielded around the latitude of $1000 \mathrm{~km}$ for energies of 1 to $10 \mathrm{MeV}$, whereas those with energies of $100 \mathrm{MeV}$ to $1 \mathrm{GeV}$ penetrate to all areas of the surface. When the incident angle is $165^{\circ}$, the protons are completely shielded around the latitude of $1000 \mathrm{~km}$ over the whole energy range of $1 \mathrm{MeV}-1 \mathrm{GeV}$.

\section{Discussion}

The results obtained in our calculation indicated that protons coming from the west were completely shielded by the magnetic anomaly, suggesting that crews would only need to focus on protons from the vertex and from the east in particular. Protons from the west are most ideally shielded by the Martian magnetic anomaly since perfect shielding can be realized over the energy range of $1 \mathrm{MeV}-1 \mathrm{GeV}$. For protons at angles of $90^{\circ}$, some radiation shielding can be expected for initial energies between 1 and $10 \mathrm{MeV}$. On the other hand, radiation exposure from protons from the east is significantly increased because these protons are concentrated on the area between the magnetic poles. It is necessary to predict the exact directions of origin of the particles since protons are rotated by the Lorentz force in the magnetic anomalies.

The characteristic distributions for incident angles of $15^{\circ}$ and $165^{\circ}$ are mainly attributable to the magnetic field perpendicular to the ground as shown in Fig. 2. In the area between latitudes of 0 and $1000 \mathrm{~km}$, the dominant component of the magnetic field is upward from the ground, and protons are turned clockwise by the Lorentz force. Conversely, the downward magnetic field is dominant in the area between latitudes of 1000 and $2000 \mathrm{~km}$, where protons are rotated counterclockwise. In addition, the Larmor radius of the protons is estimated at hundreds to thousands of kilometers for particle energies of $1 \mathrm{MeV}-1 \mathrm{GeV}$ in this magnetic anomaly. Comparing the Larmor radius with the size of the calculation area implies that the dominant motion of the proton is rotation and not drift. Therefore, protons from the west escape from the center of the magnetic anomaly, while those from the east are concentrated on the area between the magnetic poles.

Magnetic anomalies also exist at higher latitudes of the Martian southern hemisphere, where the magnetic poles are reversed against the north-south direction. It is expected that the characteristic relation between the radiation shielding and the particle concentration should be reversed here.

Since SEPs move with the interplanetary magnetic field (IMF), the actual incidences of energetic protons are expected to be different from those in this calculation. However, coronal mass ejections (CMEs) are emitted from the Sun and move in the radial direction instead of following the IMF. CMEs are the harshest solar events, posing extreme health risks owing to the significant masses involved, whose energy ranges correspond to those used in this calculation. When using magnetic anomalies to shield from CMEs, crews could be protected in the early evening, while avoiding activity at other times of day to prevent health risks. Crews could also increase their protection from such risks by inhabiting areas with adjacent natural walls to the east, such as mountains or cliffs.

Observations by NASA's Curiosity rover have revealed that the Martian atmosphere can deflect particles with energies below $150 \mathrm{MeV}$ in Gale Crater (Hassler et al., 2014). Hassler's result indicates the necessity of additional shielding for particle energies of $150 \mathrm{MeV}$ or higher. In addition, NASA and other laboratories have suggested a protection method that makes use of a magnetic dipole field at the Mars L1 Lagrange point (Green et al., 2017). This would be artificial and temporary radiation shielding that would require maintenance. On the other hand, the Martian magnetic anomaly provides natural and permanent radiation shielding without the need for maintenance, which can also cover a wider range of particle energies than the atmospheric shielding.

In summary, our numerical results show that Martian magnetic anomalies can provide complete radiation shielding against particles incident from the west but concentrate those from the east over the energy range of $1 \mathrm{MeV}-1 \mathrm{GeV}$. These results indicate that crews would only require radiation shielding against particles incident from the vertex and from the east in particular, rather than from all directions. We expect that radiation exposure to humans could be reduced effectively by using the Martian magnetic anomalies though we did not calculate their radiation doses in this study. A Martian magnetic anomaly could therefore be used as a source of continuous and efficient radiation shielding. To realize this idea, it is necessary to strengthen the level of protection against radiation incident from the east by accurately measuring the incident directions of particles in the future. Moreover, we will conduct simulations in detail including the estimation of the radiation exposure in Martian magnetic anomalies.

\section{Acknowledgement}

The computer simulation described was performed on the JAXA Supercomputer System generation 2 (JSS2) and the KDK computer system at the Research Institute for Sustainable Humanosphere, Kyoto University.

\section{References}

Acuña, M. H., Connerney, J. E. P., Wasilewski, P., Lin, R. P., Anderson, K. A., Carlson, C. W., McFadden, J., Curtis, D. W., Mitchell, D., Reme, H., Mazelle, C., Sauvaud, J. A., d'Uston, C., Cros, A., Medale, J. L., Bauer, S. J., Cloutier, P., Mayhew, M., Winterhalter, D., and Ness, N. F. (1998) Magnetic field and plasma observations at Mars: Initial results of the Mars Global Surveyor mission. Science, 279, 1676-1680.

Acuña, M. H., Connerney, J. E. P., Ness, N. F., Lin, R. P., Mitchell, D., Carlson, C. W., McFadden, J., Anderson, K. A., Rème, H., Mazelle, C., Vignes, D., Wasilewski, 
P., and Cloutier, P. (1999) Global distribution of crustal magnetization discovered by the Mars Global Surveyor MAG/ER experiment. Science, 284, 790-793.

Acuña, M. H., Connerney, J. E. P., Wasilewski, P., Lin, R. P., Mitchell, D., Anderson, K. A., Carlson, C. W., McFadden, J., Rème, H., Mazelle, C., Vignes, D., Bauer, S. J., Cloutier, P., and Ness, N. F. (2001) Magnetic field of Mars: Summary of results from the aerobraking and mapping orbits. J. Geophys. Res.: Planets, 106, 23403-23417.

Birdsall, C. K. and Langdon, A. B. (1985) Plasma physics via computer simulation. New York, McGraw-Hill.

Green, J. L., Hollingsworth, J., Brain, D., Airapetian, V., Glocer, A., Pulkkinen, A., Dong, C., and Bamford, R. (2017) A future Mars environment for science and exploration. Planetary Science Vision 2050 Workshop 2017, Washington, DC.

Hassler, D. M., Zeitlin, C., Wimmer-Schweingruber, R. F., Ehresmann, B., Rafkin, S., Eigenbrode, J. L., Brinza, D. E., Weigle, G., Böttcher, S., Böhm, E., Burmeister, S., Guo, J., Köhler, J., Martin, C., Reitz, G., Cucinotta, F. A., Kim, M., Grinspoon, D., Bullock, M. A., Posner, A., Gómez-Elvira, J., Vasavada, A., Grotzinger, J. P., and MSL Science Team. (2014) Mars' surface radiation environment measured with the Mars Science
Laboratory's Curiosity rover. Science, 343, 1244797.

Langlais, B., Purucker, M. E., and Mandea, M. (2004) Crustal magnetic field of Mars. J. Geophys. Res.: Planets, 109, E02008.

Langlais, B. and Quesnel, Y. (2008) New perspectives on Mars' crustal magnetic field. Comptes Rendus Geosci., 340, 791-800.

Mitchell, D. L., Lillis, R. J., Lin, R. P., Connerney, J. E. P., and Acuña, M. H. (2007) A global map of Mars' crustal magnetic field based on electron reflectometry. $J$. Geophys. Res.: Planets, 112, E01002.

Musk, E. (2017) Making humans a multi-planetary species. New Space, 5, 46-61.

Purucker, M., Ravat, D., Frey, H., Voorhies, C., Sabaka, T., and Acuña, M. (2000) An altitude-normalized magnetic map of Mars and its interpretation. Geophys. Res. Lett., 27, 2449-2452.

Sakurai, K. (1974) Physics of solar cosmic rays. Tokyo, University of Tokyo Press.

Smith, R. M., Merancy, N., and Krezel, J. (2017) NASA planning for exploration mission 2 (EM-2) and accomplishing exploration objectives in the proving ground. 2017 IEEE Aerosp. Conf., pp. 1-12, Big sky. 